\title{
Comparison of Mortality by Gender and Regions in Tanzania using Direct Standardized Death Rates (DSDR) Method
}

\author{
${ }^{1}$ shadrack Elia Kibona ${ }^{\S},{ }^{2}$ christopher Hariri Mbotwa \\ ${ }^{1}$ Department of Statistics, University of Dar es Salaam, Tanzania \\ ${ }^{2}$ University of Dar es Salaam, Mbeya College of Health and Allied Sciences, Tanzania
}

\begin{abstract}
Background: Comparison of mortality is very useful in assessing the population health. Crude rates can easily be computed from the mortality but they are not good for comparison across groups of the population. The aim of this paper was to compare mortality in Tanzania by region and gender using 2012 Tanzania Population and Housing Census.

Methods: Age-specific death rates for regions, Tanzania Mainland, Tanzania Zanzibar and entire Tanzania were obtained from Mortality and Health monograph data downloadable from the National Bureau of Statistics website. The direct standardization method was used to compare the mortality for male and female populations across all regions of Tanzania Mainland and Zanzibar.

Results: Findings show that the mortality is low in Arusha, Manyara and Kilimanjaro compared to other regions in Tanzania mainland implying that health status for both male and female population in those regions is better than the other regions while it is the worst in Njombe, Iringa, and Kagera implying the poor health status for those regions as compared to the rest regions in Tanzania Mainland. In Tanzania Zanzibar, high mortality was observed in Kusini Unguja and the lowest in Kaskazini Unguja for both male and female populations. By national wise and for almost all regions, the mortality for male population is higher than that for female population.

Conclusion: Direct standardization methods can save as the best way for comparing mortality because it takes into consideration both the population at risk and the age structure. However, in estimating mortality, crude death rates should be used to give the magnitude while direct standardized death rates should be used for comparison purposes.

Keywords: Age, Crude, Death, Mortality, and Standardized
\end{abstract}

\section{Introduction}

In the year 2015, Africa was characterized by high mortality risk at all ages while the world mortality at old ages that is sixty five and above years recorded 55 percent compared to 41 percent in 1990 contributed by rise in percent of deaths in Asia, Latin America, and the Caribbean (United Nations, 2017). In order to assess progress and health improvement in the world, estimation of mortality is of important (Wang et al., 2012). Likewise, one can make a decision in allocation of scarce resources among various regions in a specific country by comparing mortality indicators as well other health events.

Comparison of mortality by geographical areas or by other socio-economic characteristics is very useful in assessing population health (Julious et al., 2001). In most cases the absolute rate is usually not good for comparison or for assessing mortality in the population over a specified period of time. This is because the absolute rates depend on size of the population regardless of the population at risk. Thus, it is very important to compute summary statistics which take care of the variation in age structure (Ahmad et al., 2001).

Crude death rate (CDR) and Age-specific death rate (ASDR) are popular indicators computed for expressing mortality in a specified area and time. These methods are simple in computation but face challenges when one tries to compare mortality between two or more countries or regions. This

§Corresponding e-mail: cmbotwa@gmail.com 
Volume 21, Number 1, April 2019

is because CDR assumes that the entire population is exposed to the same risk of dying. But in reality, deaths tend to vary from one population to another depending on the underlying age structure. For example, aging population can have a high CDR even if the health status of the population is good. Though, the ASDR expresses the death rates for each age group, it is not suitable for comparing mortality of two or more population because of the difference in age structure. Thus, in order to take care of the difference in the age structure of two or more population, some methods of standardization which adjusts for the difference in age structure must be used. There are various methods of comparing mortality among them are direct and indirect standardization (Wolfenden, 1923). However, with the availability of data on age-specific death rate, direct standardized methods become dominant in areas of demography and epidemiology (Ahmad et al., 2001).

Crude Death rate expresses the number of deaths in a particular country or area and time over the mid-year population per thousand.

$C D R=\frac{\text { Number of Deaths in a particular Year }}{\text { Midyear Population }} * 1000$

This rate does not take care of the difference in exposure to death risk. Therefore, in assessing the health status of two or more population, CDR may not be suitable measure. For example, demographic analysis shows that for a particular cohort, as age increase to the highest the probability of dying also increase approaching one. High mortality in a country with the aging population for which most of the deaths occur in the higher age is not as bad as a country with high mortality which is contributed by deaths in age groups rather than higher age (Christensen, Doblhamme, Rau, \& Vaupel, 2009).

According to 2012 Tanzania Population and Housing Census (TPHC), the CDR for male's population in Tanzania was 10.0 per thousand population and for females was 8.6 per thousand population with the country`s CDR of 9.3 per thousand population. From these indicators, we cannot directly conclude that males are at high risk of death compared to females because the rate includes all individuals regardless of their exposure to the risk of death while practically, males and female experiences difference risks of death through different age groups which is not taken care by CDR.

Table 1: CDR by Gender across different Regions of Tanzania as per 2012 TPHC

\begin{tabular}{lccc}
\hline Region/Area & \multicolumn{3}{c}{ 2012 Estimated CDRs } \\
\cline { 2 - 4 } & Total & Male & Female \\
\hline Tanzania & 9.3 & $\mathbf{1 0 . 0}$ & $\mathbf{8 . 6}$ \\
Tanzania Mainland & 9.4 & $\mathbf{1 0 . 1}$ & $\mathbf{8 . 6}$ \\
Dodoma & 9.0 & 10.1 & 7.9 \\
Arusha & 5.4 & 5.9 & 4.9 \\
Kilimanjaro & 9.3 & 9.4 & 9.2 \\
Tanga & 9.1 & 9.6 & 8.6 \\
Morogoro & 9.2 & 10.0 & 8.5 \\
Pwani & 12.2 & 12.3 & 12.2 \\
Dar es Salaam & 9.1 & 10.2 & 8.1 \\
Lindi & 10.2 & 11.5 & 9.0 \\
Mtwara & 10.6 & 11.2 & 10.0 \\
Ruvuma & 9.9 & 10.5 & 9.3 \\
Iringa & 12.5 & 13.0 & 11.9 \\
Mbeya & 10.7 & 11.4 & 10.1 \\
Singida & 7.8 & 8.1 & 7.4 \\
Tabora & 9.5 & 10.5 & 8.4
\end{tabular}




\begin{tabular}{lccc}
\hline Region/Area & \multicolumn{3}{c}{ 2012 Estimated CDRs } \\
\cline { 2 - 4 } & Total & Male & Female \\
\hline Rukwa & 10.1 & 11.2 & 9.0 \\
Kigoma & 9.6 & 10.2 & 9.0 \\
Shinyanga & 9.7 & 10.7 & 8.7 \\
Kagera & 11.4 & 12.1 & 10.7 \\
Mwanza & 8.4 & 9.2 & 7.6 \\
Mara & 9.5 & 10.1 & 8.9 \\
Manyara & 6.9 & 7.4 & 6.5 \\
Njombe & 13.5 & 14.9 & 12.4 \\
Katavi & 10.7 & 12.3 & 9.0 \\
Simiyu & 7.4 & 7.8 & 7.1 \\
Geita & 7.6 & 8.3 & 6.9 \\
Tanzania Zanzibar & 7.0 & 7.9 & 6.2 \\
Kaskazini Unguja & 7.1 & 8.2 & 6.1 \\
Kusini Unguja & 9.0 & 9.9 & 8.1 \\
Mjini Magharibi & 6.5 & 7.6 & 5.6 \\
Kaskazini Pemba & 7.0 & 7.8 & 6.2 \\
Kusini Pemba & 7.4 & 7.7 & 7.2 \\
\hline Source: NBS, 2015 & & & \\
\hline
\end{tabular}

Source: NBS, 2015

CDRs for individual age cohorts, called age-specific death rates (ASDRs), are the ratio of the number of deaths in a given age group to the population of that age group, and usually expressed per 1,000 populations. ASDR can be used as an alternative for CDR because it gives the rates for each age group thereby allowing the comparison among different populations.

$$
\text { Age }- \text { Specific Death Rate }=\frac{\text { Number of Deaths in Specified Age group }}{\text { Number of Persons in that Specified Age group }} X 1000
$$

ASDR is one of the comprehensive and reliable methods of comparing death rates over time or between different population groups because it compares individual's death rates for all age groups of population of interest. However, the method requires too many comparisons and hence become tiresome for both researcher and targeted audience.

Because the CDR is not good for comparison and ASDRs gives too much detailed information, a standardized statistical measure that takes care for a population's age distribution should be used (Shyrock \& Siegel, 1971; Spiegelman M, 1968).The two main common types of standardized rates are Direct Standardized Rate (DSDR) and Indirect Standardized Death Rate (ISDR).

Direct standardization results to age-adjusted death rate, which is a weighted average of the age-specific death rates, for the populations to be compared (Ahmad et al., 2001). Here the weights represent the relative age distribution of the standard population hence, producing a single summary for each population which reflects the number of deaths that would have been happened if the populations being compared had had the same age distribution. Indirect standardization method considers the averages of death rates for each specific population with reference to the chosen standard population. The method is used when the ASDRs of the study Population are not known with population proportions in each age group.

The choice of standardization method (direct or indirect) depends on the availability of the data for the age-specific death rates. Therefore, this paper employed a direct standardization method to compare mortality between males and females across regions of Tanzania Mainland and Zanzibar. The formula for arriving at DSDRs is presented by the following mathematical equation. 
$D S D R=\frac{\sum r_{i} N_{i}}{\sum N_{i}}$

Where, $r_{i}$ is the age-specific death rate in the $i^{\text {th }}$ age group and $N_{i}$ is the number of persons in the $i^{\text {th }}$ age group.

\section{Methods and Materials}

ASDRs were constructed from abridged life tables based on deaths and population as per 2012 TPHC published in the Mortality and Health Monograph report (2015) by National Bureau of Statistics (NBS). The population for each age group was obtained from Age and Sex Distribution Report (2013) produced from 2012 TPHC. When constructing DSDRs for Tanzania mainland and Tanzania Zanzibar, the Tanzania population was used as a standard population. This allowed a comparison between males and females across Tanzania Mainland and Tanzania Zanzibar.

For computations of DSDRs for regions in Tanzania, Tanzania Mainland population was used as the standard population and likewise for regions in Tanzania Zanzibar, the Tanzania Zanzibar population was used as the standard population. This enabled the researcher to make comparison of mortality between males and females in Tanzania Mainland and Tanzania Zanzibar separately. When constructing the ASDRs, the age group $80+$ was omitted because the probability of dying for a person aged 80 years and above is very high implying that persons aged 80 years will eventually die. In practice the survivorship to infinity years of age is impossible because human being must eventually die. The results were then tabulated for males and females across all regions of Tanzania.

\section{Results and Discussion}

The findings show that DSDR in Tanzania Mainland was 3.4 and 2.8 per 1,000 population for males and females respectively; while for Tanzania Zanzibar, DSDR was 2.7 and 2.1 per 1,000 population for males and females respectively. These finding imply that, for both Tanzania

Table 2: Direct Standardized Death Rates by Gender across Regions in Tanzania as per 2012 TPHC

\begin{tabular}{lcccc}
\hline Region & Male & $\begin{array}{c}\text { Rank in Tanzania } \\
\text { Mainland/Zanzibar }\end{array}$ & Female & $\begin{array}{c}\text { Rank in Tanzania } \\
\text { Mainland/Zanzibar }\end{array}$ \\
\hline Tanzania Mainland & 3.4 & N/A & 2.8 & N/A \\
Dodoma & 3.2 & 16 & 2.0 & 23 \\
Arusha & 1.9 & 25 & 1.4 & 25 \\
Kilimanjaro & 2.3 & 23 & 2.1 & 22 \\
Tanga & 2.7 & 21 & 2.5 & 18 \\
Morogoro & 3.2 & 14 & 2.6 & 14 \\
Pwani & 3.3 & 12 & 3.3 & 5 \\
Dar Es Salaam & 3.8 & 8 & 3.1 & 9 \\
Lindi & 3.0 & 18 & 2.3 & 20 \\
Mtwara & 2.9 & 19 & 2.5 & 17 \\
Ruvuma & 3.5 & 10 & 3.2 & 8 \\
Iringa & 4.6 & 2 & 3.8 & 2 \\
Mbeya & 4.0 & 6 & 3.5 & 3 \\
Singida & 2.4 & 22 & 2.1 & 21 \\
Tabora & 3.7 & 9 & 2.8 & 12
\end{tabular}




\begin{tabular}{lcccc}
\hline Region & Male & $\begin{array}{c}\text { Rank in Tanzania } \\
\text { Mainland/Zanzibar }\end{array}$ & Female & $\begin{array}{c}\text { Rank in Tanzania } \\
\text { Mainland/Zanzibar }\end{array}$ \\
\hline Rukwa & 4.1 & 5 & 3.2 & 6 \\
Kigoma & 3.2 & 15 & 2.7 & 13 \\
Shinyanga & 3.9 & 7 & 3.0 & 10 \\
Kagera & 4.1 & 4 & 3.4 & 4 \\
Mwanza & 3.3 & 13 & 2.6 & 15 \\
Mara & 3.5 & 11 & 2.9 & 11 \\
Manyara & 2.2 & 24 & 1.9 & 24 \\
Njombe & 5.7 & 3 & 4.2 & 1 \\
Katavi & 4.5 & 20 & 3.2 & 7 \\
Simiyu & 2.8 & 17 & 2.4 & 19 \\
Geita & 3.0 & NA & 2.6 & 16 \\
Tanzania Zanzibar & 2.7 & 4 & $\mathbf{2 . 1}$ & NA \\
Kaskazini Unguja & 2.4 & 1 & 1.7 & 5 \\
Kusini Unguja & 3.1 & 2 & 2.3 & 1 \\
Mjini Magharibi & 2.7 & 4 & 1.9 & 3.5 \\
Kaskazini Pemba & 2.4 & 4 & 1.9 & 3.5 \\
Kusini Pemba & 2.4 & 2.1 & 2 \\
\hline
\end{tabular}

Mainland and Zanzibar, mortality is high for male population as compared to that of female population which give the same implications as the results of CDR which also show high mortality for males (10.1 deaths per 1000 population) than for females (8.6 deaths per 1000 population). Thus, the finding suggests that by taking into consideration the population at risk of death and by standardizing the age-sex structure, there were more deaths among males as compared to females. Except for Pwani region, all other regions in both Tanzania Mainland and Tanzania Zanzibar had high mortality for male population than for female population.

The ranking of region in Table 2 in descending order of the DSDRs' magnitude is the best way of comparing mortality by areas with reference to the standard population. This approach is is very useful in comparing the incidence of mortality across different regions of Tanzania Mainland and Tanzania Zanzibar. By standardizing each region's population age structure to Tanzania mainland age structure, regions leading in high mortality are: Njombe and Iringa for both male and females. For male population, other regions following Njombe and Iringa are Katavi,Kagera, Rukwa, Mbeya, Shinyanga, Dar es Salaam, Tabora, Ruvuma, Mara, Pwani, Mwanza, Morogoro, Kigoma, Dodoma, Geita, Lindi, Mtwara, Simiyu, Tanga, Singida, Kilimanjaro, Manyara and Arusha in a decreasing order of DSDRs. while for female population the DSDRs are arranged in decreasing order from Njombe, Iringa, Mbeya, Kagera, Pwani, Rukwa, Katavi, Ruvuma, Dar es Salaam, Shinyanga, Mara, Tabora, Kigoma, Morogoro, Mwanza, Geita, Mtwara, Tanga, Simiyu, Lindi, Singida, Kilimanjaro, Dodoma, Manyara, to Arusha in decreasing order of DSDRs. these findings show a little difference with those presented in the Mortality and Health Monograph by (NBS, 2015) whereby Njombe Pwani and Iringa had the highest crude death rate while using standardized death rate, Pwani is not among the three regions with the highest mortality. Generally, looking at the rankings for DSDRs for males and females, the results indicate that male population has high mortality as compared to female population because for each rank the DSDRs for males is higher than that for females. 
These findings indicate that the health statuses in regions of Njombe, Iringa, Kagera, and Mbeya are the worst (because of high DSDRs for both male and female population) and on the other hand; Arusha, Manyara, and Kilimanjaro have had better health status compared to other regions because their DSDRs are very small for both male and female populations. The highest Mortality in Njombe, Iringa, and Mbeya might be due to high HIV prevalence in those regions; 11.4 percent, 11.3 percent and 9.3 percent respectively (NBS, 2017). While in Kagera with the high mortality might be due to high Malaria prevalence(NBS \& ICF, 2016). Thus, high Malaria prevalence in Kagera and high HIV prevalence in Njombe, Iringa and Mbeya be might the the causes of high death rates hence raising the mortality level. For the case of Arusha, Kilimanjaro and Manyara, the findings are in line with statistics from the 2012 census which show that Arusha, Kilimanjaro and Manyara regions had the lowest crude death rate and Infant mortality rates (NBS, 2015).

On the other hand, for Tanzania Zanzibar, Kusini Unguja were found to have the highest mortality for both male and female populations (DSDRs 3.1 and 2.3 for male and female population respectively). This implies that the health status of Kusini Unguja is poor compared to other regions in Tanzania Zanzibar. Kaskazini Unguja had had the lower DSDRs for female population and for male population, the lowest DSDRs were observed in Kasikazini Unguja, Kaskazini Pemba, and Kusini Pemba. Thus, Kasikazini Unguja has had a good health status compared to other regions in Tanzania Zanzaibar.

\section{Conclusion and Recommendation}

For better results of comparing mortality across difference regions, CDR should not be used because it does not take into consideration the age structure of the underlying populations. Although ASDRs take into consideration the age structure of the population, the method gives too many information hence creates a difficult for researchers and readers when trying to make comparison of mortality over different regions. Theretofore, given the availability of ASDRs data, Direct Standardization method could save as one of the best methods for comparisons of mortality for different populations. This method gains the credits by overcoming the drawbacks of the other methods (CDRs and ASDRs). However, for better estimation of mortality, all these methods should be used together because the figures obtained by standardization methods cannot be used to indicate the magnitude of mortality. They can only be used for comparison purposes. The magnitude of the mortality should always be obtained by CDR method. The government and Non-governmental organizations should embark on reducing risk factors associated with mortality in regions ranked high compared to others. The government should increase its budget on improving health sectors to reduce mortality for all age groups and provide sensitization on risk factors to mortality like HIV in those regions ranked high mortality at national level. These findings can guide proper allocation of funds given limited resources. This study compared mortality for gender and regions of Tanzania. There is a potential of other studies to compare the mortality with other countries as well as by other socio-economic status.

\section{Conflict of interests: Authors declare no conflict of interests}

\section{References}

Ahmad, O. B., Boschi-Pinto, C., Lopez, A. D., Murray, C. J., Lozano, R., \& Inoue, M. (2001). Age Standardization of Rates: A new WHO Standard, 14.

Christensen, K., Doblhamme, G., Rau, R., \& Vaupel, J. W. (2009). Ageing populations: the challenges ahead. Retrieved 8 August 2018, from https://www.ncbi.nlm.nih.gov/pmc/articles/PMC2810516/

Julious, S. A., Nicholl, J., \& George, S. (2001). Why do we continue to use standardized mortality ratios for small area comparisons? Journal of Public Health, 23(1), 40-46.

Mageda, K., Leyna, G. H., \& Mmbaga, E. J. (2012). High initial HIV/AIDS-Related mortality and-its predictors among patients on antiretroviral therapy in the kagera region of Tanzania: a five-year retrospective cohort study. AIDS Research and Treatment, 2012. 
National Bureau of Statistics (NBS) [Tanzania]. (2015). Mortality and Health Monograph. $\quad$ Dar es Salaam, Tanzania.

National Bureau of Statistics (NBS) [Tanzania]. (2017). Tanzania HIV Impact Survey (THIS). $\quad$ Dar es Salaam, Tanzania.

National Bureau of Statistics (NBS) [Tanzania] and ICF Macro. (2016). Tanzania Demographic and Health Survey and Malaria Indicator Survey (TCHS-MIS) 2015-16. Dar es Salaam, Tanzania: NBS and ICF Macro.

Shyrock, H. S., \& Siegel, J. S. (1971). The methods and materials of demography (Vol. 2). U.S, Bureau of the Census, Washington: Government Printing Office.

Spiegelman M. (1968). Introduction to demography (Rev. ed.). Cambridge: Harvard University Press.

United Nations. (2017). World Mortality 2017: Data Booklet.

Wang, H., Dwyer-Lindgren, L., Lofgren, K. T., Rajaratnam, J. K., Marcus, J. R., Levin-Rector, A., ... Murray, C. J. (2012). Age-specific and sex-specific mortality in 187 countries, 1970-2010: a systematic analysis for the Global Burden of Disease Study 2010. The Lancet, 380(9859), 2071-2094.

Wolfenden, H. H. (1923). On the methods of comparing the moralities of two or more communities, and the standardization of death-rates. Journal of the Royal Statistical Society, 86(3), 399-411. 